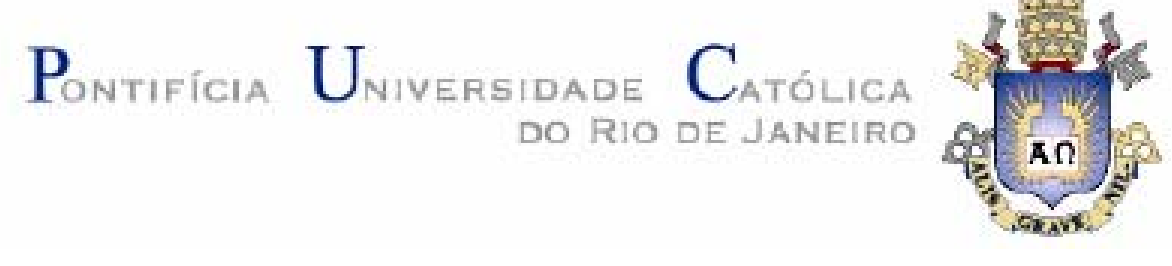

Antonio Eduardo Gonçalves Sampaio

\title{
Análise do Comportamento Dinâmico de Colunas Semi-Enterradas
}

Dissertação de Mestrado

Dissertação apresentada como requisito parcial para obtenção do título de Mestre pelo Programa de PósGraduação em Engenharia Civil da PUC-Rio. Área de Concentração: Estruturas.

Orientador: Prof. Paulo Batista Gonçalves

Departamento de Engenharia Civil

Pontifícia Universidade Católica

Rio de Janeiro, setembro de 2004 


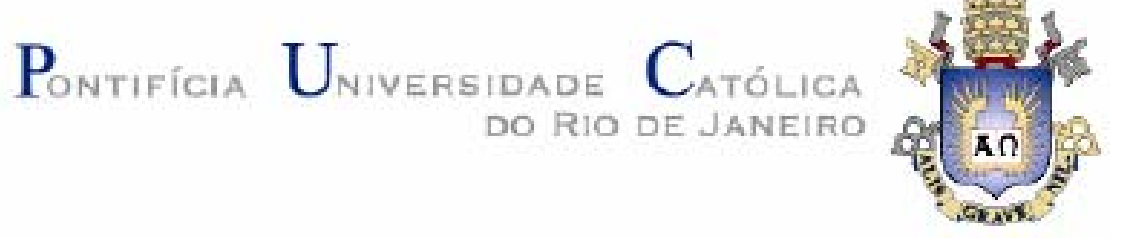

Antonio Eduardo Gonçalves Sampaio

\section{Análise do Comportamento Dinâmico de Colunas Semi-Enterradas}

Dissertação apresentada como requisito parcial para obtenção do título de Mestre pelo Programa de PósGraduação em Engenharia Civil da PUC-Rio. Aprovada pela Comissão Examinadora abaixo assinada.

Prof. Paulo Batista Gonçalves

Presidente/Orientador Departamento de Engenharia Civil - PUC-Rio

Prof. José Guilherme Santos da Silva Universidade do Estado do Rio de Janeiro - UERJ

Prof. João Luis Pascal Roehl Departamento de Engenharia Civil - PUC-Rio

Prof. Raul Rosas e Silva Departamento de Engenharia Civil - PUC-Rio

Prof. José Eugênio Leal

Coordenador Setorial do Centro Técnico Científico - PUC-Rio

Rio de Janeiro, 03 de Setembro de 2004 
Todos os direitos reservados. É proibida a reprodução total ou parcial do trabalho sem autorização da universidade, do autor e do orientador.

\section{Antonio Eduardo Gonçalves Sampaio}

Graduou-se em Engenharia Civil com ênfase em estruturas em 2001 na PUC-Rio. Atualmente, trabalha como Engenheiro Civil em Furnas Centrais Elétricas S.A.

Ficha Catalográfica

Sampaio, Antonio Eduardo Gonçalves

Análise do comportamento dinâmico de colunas semienterradas / Antonio Eduardo Gonçalves Sampaio ; orientador: Paulo Batista Gonçalves. - Rio de Janeiro : PUC-Rio, Departamento de Engenharia Civil, 2004.

$121 \mathrm{f.}:$ il. ; $30 \mathrm{~cm}$

Dissertação (mestrado) - Pontifícia Universidade Católica do Rio de Janeiro, Departamento de Engenharia Civil .

Inclui referências bibliográficas

1. Engenharia Civil - Teses. 2. Colunas. 3. Estacas. 4. Vibrações. 5. Fundação elástica. I. Sampaio, Antonio Eduardo Gonçalves. II. Pontifícia Universidade Católica do Rio de Janeiro. Departamento de Engenharia Civil . III. Título. 
Aos meus pais, Antonio Sampaio e Maria de Lourdes.

À minha irmã Rejane e demais familiares. 


\section{Agradecimentos}

À PUC-Rio e aos professores do departamento pela oportunidade.

Ao Prof. Paulo Batista Gonçalves pelo apoio, incentivo, serenidade e paciência.

À banca examinadora.

Aos meus amigos de longa data Alexandre Vale, Fernando Arraes e Yuri Arraes pela convivência fora do ambiente da Universidade.

Aos colegas que apoiaram algumas etapas desta Dissertação em especial a Gustavo Serebrenick, Eduardo Pasquetti, Frederico Martins e Alexandre Pereira.

Aos funcionários e aos demais colegas de Mestrado.

À CAPES pelo auxílio financeiro que viabilizou este trabalho. 


\section{Resumo}

Sampaio, Antonio Eduardo Gonçalves; Gonçalves, Paulo Batista. Análise do comportamento dinâmico de colunas semi-enterradas. Rio de Janeiro, 2004. 121p. Dissertação de Mestrado - Departamento de Engenharia Civil, Pontifícia Universidade Católica do Rio de Janeiro.

O estudo de estacas semi-enterradas tem grande importância na construção civil devido principalmente à sua ampla aplicação em diversas áreas da engenharia. O presente trabalho tem como objetivo obter uma solução exata para as freqüências naturais e modos de vibração dessas estacas e, com base nestes resultados, analisar o comportamento do sistema não-linear. A análise das vibrações lineares parte da obtenção de um funcional de energia e dedução das equações diferenciais parciais de movimento para o trecho enterrado e desenterrado e a determinação das diversas famílias de soluções para cada equação de movimento em função dos parâmetros adimensionais característicos do problema. Essa solução exata possibilita mostrar a influência dos parâmetros de carregamento, de rigidez da fundação, da altura da fundação e das condições de apoio nas freqüências naturais e modos de vibração. A não-linearidade do problema é originária da geometria da estrutura e a sua consideração implica na obtenção de equações diferenciais não-lineares. A resolução destas equações é feita de forma aproximada, empregando-se o método de Ritz para discretizar a coluna no espaço e os métodos de Galerkin-Urabe e do Balanço Harmônico para resolver as equações de movimento resultantes do processo de discretização. Com base nestes resultados, analisa-se a influência dos parâmetros físicos e geométricos do sistema coluna-fundação nas vibrações livres e forçadas da estaca.

\section{Palavras-chave}

Colunas, fundação elástica, vibrações, estacas. 


\section{Abstract}

Sampaio, Antonio Eduardo Gonçalves; Gonçalves, Paulo Batista (Advisor). Analysis of the dynamic behavior of semi-embedded columns. Rio de Janeiro, 2004, 121p. MSc. Dissertation - Department of Civil Engineering, Pontifícia Universidade Católica do Rio de Janeiro.

The study of partially embedded piles is an important topic in civil engineering due to its use in several engineering fields. The aim of the present work is to derive an exact solution for the natural frequencies and vibration modes of these piles and, based on these results, to analyze the nonlinear behavior of the column-foundation system. In order to study the free vibration problem, the energy functional of the system is obtained, allowing the derivation of the partial differential equations of motion for the buried and unburied parts of the pile. The possible solutions of these equations are then obtained as a function of a set of non-dimensional parameters characteristic of the problem. The consideration of the geometric nonlinearity of the beam leads to a set of nonlinear differential equations. The solution of these equations is obtained by employing the Ritz method to discretize the column in space and the methods of Galerkin-Urabe and Harmonic Balance to derive an approximate solution for the resulting equations of motion. Based on these results, the influence of the geometrical and physical parameters of the column-foundation system on the free and forced non-linear vibrations of the pile is analyzed.

\section{Keywords}

Columns, elastic-foundation, vibrations, piles. 


\section{Sumário}

1 Introdução 17

1.1. Motivação 19

1.2. Objetivos 19

1.3. Organização do Trabalho 20

2 Formulação do Problema 21

2.1. Funcional de Energia Não-Linear 21

2.1.1. Energia Potencial Total 23

2.1.2. Fundação Elástica 26

2.1.3. Energia Cinética 27

2.1.4. Amortecimento 27

2.1.5. Força Harmônica 28

2.1.6. Montagem do Funcional de Energia Não-Linear 29

2.2. Funcional de Energia Linear 29

3 Freqüências Naturais e Modos de Vibração 31

3.1. Solução Analítica do Problema de Autovalor 31

3.2. Solução Aproximada de Rayleigh-Ritz 39

4 Resultados Numéricos $\quad 41$

4.1. Obtenção dos parâmetros da Base Elástica e de Carga 41

4.2. Obtenção do Parâmetro da Freqüência Natural pelo Método de Rayleigh-Ritz 47

4.3. Influência dos Parâmetros da Base Elástica nos Valores do Parâmetro da Freqüência Natural 48

4.4. Influência da Profundidade da Fundação 54

4.5. Influência do Carregamento - Parâmetro de Carga $\lambda \quad 60$

4.6. Influência das Condições de Contorno 63 
5 Análise Não-Linear pelos Métodos de Galerkin-Urabe e Balanço Harmônico 74

5.1. Método de Galerkin-Urabe 76

5.2. Método do Balanço Harmônico 77

5.3. Resolução do Sistema de Equações Não-Lineares por Newton-Rapson 79

6 Resultados da Análise Não-Linear 81

6.1. Relação Freqüência e Amplitude (Vibração Livre) 81

6.2. Vibração Forçada - Ressonância Não-Linear e 85 Bifurcações

7 Conclusões e Sugestões $\quad 95$

8 Referências Bibliográficas 98

9 Apêndice 101

9.1. Análise Linear 101

9.2. Análise Não-Linear 115 


\section{Lista de Figuras}

Figura 2.1: Deslocamento transversal e encurtamento da vigacoluna.

Figura 2.2: Deslocamento da linha neutra.

Figura 2.3: Representação da profundidade da fundação.

Figura 3.1: Trechos desenterrado (1) e enterrado (2) da coluna parcialmente enterrada.

Figura 3.2: Condições de contorno homogêneas da coluna. 37

Figura 3.3: Ponto de continuidade P.

Figura 4.1: Influência de $K$ nos valores de $\lambda_{\text {crit }}$ para diferentes condições de contorno.

Figura 4.2: Comprimentos $L_{s}$ e $L$ da estaca totalmente enterrada.

Figura 4.3: Parâmetro de freqüência versus rigidez $K$ da fundação pelo método de Rayleigh-Ritz.

Figura 4.4: Variação do parâmetro da freqüência em função do parâmetro de rigidez da fundação.

Figura 4.5: Modos de vibração para $K=500$. 50

Figura 4.6: Modos de vibração para $K=2.000$. 51

Figura 4.7: Modos de vibração para $K=20.000$. 52

Figura 4.8: Modos de vibração para $K=75.000$. 53

Figura 4.9: Influência da profundidade $h$ da fundação nos valores do parâmetro das freqüências naturais para $K=2.000$.

Figura 4.10: Influência da profundidade $h$ da fundação nos valores do parâmetro das freqüências naturais para $K=20.000$ e $K=75.000$. 
Figura 4.11: Relação $\Omega \times n$ para o caso em que $K=2.000$ e a coluna encontra-se totalmente enterrada.

Figura 4.12: Superposição das curvas das duas primeiras freqüências de vibração para diferentes valores de K.

Figura 4.13: Modos de vibração para diferentes profundidades da fundação.

Figura 4.14: Variação do quadrado da menor freqüência de vibração versus a porcentagem do parâmetro de carga para 5 valores diferentes de $K$.

Figura 4.15: Variação do quadrado da freqüência versus a porcentagem do parâmetro de carga para 5 valores diferentes da profundidade da fundação.

Figura 4.16: Variação do quadrado da freqüência versus a porcentagem do parâmetro de carga para 2 valores diferentes de $K$.

Figura 4.17: Modos de Vibração para a situação 3 e $K=500$, considerando cinco profundidades para a fundação.

Figura 4.18: Modos de Vibração para a situação 5 e $K=500$, considerando cinco profundidades para a fundação.

Figura 4.19: Modos de Vibração para a situação 6 e $K=500$, considerando cinco profundidades para a fundação.

Figura 4.20: Modos de Vibração para a situação 3 e $K=20.000$, considerando cinco profundidades para a fundação.

Figura 4.21: Modos de Vibração para a situação 5 e $K=20.000$, considerando cinco profundidades para a fundação.

Figura 4.22: Modos de Vibração para a situação 6 e $K=20.000$, considerando cinco profundidades para a fundação.

Figura 4.23: Coluna semi-enterrada de extremidade inferior livre submetida à carga $P$.

Figura 4.24: Comparação entre os modos de Vibração para $K=$ $500, K=20.000$ e $h=0,25$. 
Figura 4.25: Comparação entre os modos de Vibração para $K=$ $500, K=20.000$ e $h=0,5$.

Figura 4.26: Evolução dos deslocamentos, ao longo da coluna, dos primeiros modos de vibração para profundidades crescentes.

Figura 6.1: Variação da amplitude $A_{1}$ da relação $\Omega / \Omega_{\mathrm{n}}$ para os casos em que $K=500$ e $K=20.000$ com carregamento variável.

Figura 6.2: Variação da amplitude $A_{1}$ pela relação $\Omega / \Omega_{\mathrm{n}}$ para os casos em que $K=500$ e $K=20.000$ com a profundidade variável.

Figura 6.3: Relação $\Omega / \Omega_{\mathrm{n}}$ pela amplitude $A_{1}$ para cinco valores de $A_{0}$ e $K=500$.

Figura 6.4: Fenômeno do salto para a coluna semi-enterrada submetida à vibração forçada sem amortecimento.

Figura 6.5: Variação das amplitudes $A_{1}$ e $A_{2}$ versus a razão $\Omega / \Omega_{\mathrm{n}}$ para três profundidades de fundação, nos casos em que $K=500$.

Figura 6.6: Variação das amplitudes $A$ versus a razão $\Omega / \Omega_{n}$.

Figura 6.7: Comportamento das amplitudes $A_{1}$ e $A_{2}$ da estaca, para determinadas porcentagens da carga crítica aplicadas.

Figura 6.8: Comportamento da amplitude $A$ da estaca, para duas parcelas da carga crítica aplicadas.

Figura 6.9: Relação das amplitudes $A_{1}$ e $A_{2}$ versus o parâmetro de freqüência normalizado para fatores de amortecimento diferenciados. 


\section{Lista de Tabelas}

Tabela 4.1: Valores de $\mathrm{k}_{\mathrm{s} 1}$ em $\mathrm{t} / \mathrm{ft}^{3}$ para uma placa quadrada e solo argiloso sobreadensado, obtidos em Poulos (1980).

Tabela 4.2: Típicos valores de $n_{h}$, em t/ft ${ }^{3}$, para diversos níveis de adensamento de areia, obtidos em Poulos (1980).

Tabela 4.3: Valores de $k_{0}$ e de $\mathrm{K}$ para diversos tipos de solos.

Tabela 4.4: Valores de $\lambda_{\text {crit }}$ para diferentes condições de contorno. 


\section{Lista de Símbolos}

A, área da seção transversal da estaca; matriz dos coeficientes de um sistema de equações; raiz quadrada da soma do quadrado das amplitudes $A_{1}$ e $A_{2} ;$

$A_{0}, \quad$ amplitude da carga harmônica senoidal $P(t)$;

$A_{1}, \quad$ amplitude de oscilação relacionada à função seno;

$A_{2}, \quad$ amplitude de oscilação relacionada à função co-seno;

E, módulo de elasticidade ou módulo de Young;

$H$, altura da fundação;

I, momento de inércia da seção transversal;

$K$, parâmetro adimensional de rigidez da base elástica;

L, comprimento da estaca;

$L_{g}$, função de Lagrange;

$L_{\text {min }} \quad$ comprimento total mínimo para que a estaca tenha um ponto intermediário de engaste;

$L_{s}, \quad$ profundidade do ponto de engastamento intermediário;

$M$, massa da estaca;

$P, \quad$ carga axial aplicada no topo da estaca;

$P(t)$, carga harmônica senoidal;

$P_{1}, \quad$ ponto intermediário da estaca quando esta se encontra em seu estado natural;

$P_{2}$, ponto intermediário da estaca quando esta se encontra sob a ação da carga $P$;

$R$, parâmetro definido pelo critério de Davisson \& Robinson;

$\frac{1}{R}$, raio de curvatura da estrutura indeformada;

$\frac{1}{\bar{R}}, \quad$ raio de curvatura do eixo deformado;

$R e, \quad$ trabalho realizado pela força de amortecimento; 
T, energia cinética;

$U$, energia interna de deformação;

$U_{f d}$, energia interna de deformação da fundação elástica;

$V_{p}, \quad$ potencial das cargas externas;

$W_{n c}$, trabalho realizado por forças não conservativas;

$W_{p}$, trabalho realizado pela carga harmônica;

$a_{i j}$, elemento da matriz $A$ localizado na linha $i$ e na coluna $j$;

$a_{j}, \quad$ coeficiente da função j do método de Rayleigh-Ritz;

$a_{n}, \quad$ raiz do polinômio característico;

c, $\quad$ coeficiente que multiplica $x$ em uma função $f(x)$;

$c(t)$, amplitude do deslocamento em função do tempo;

d, largura da coluna;

$d s$, elemento curvo;

$d x$, elemento linear na direção do eixo axial $\mathrm{x}$;

$f_{n}$, função de aproximação do método de Rayleigh-Ritz;

$h$, comprimento adimensional da estaca;

$k_{h}$ modulo de reação da base elástica;

$\bar{k}_{s 1}$, módulo para uma placa horizontal quadrada de $1 \mathrm{ft}$ de lado;

$k_{0}, \quad$ coeficiente de rigidez da fundação; número associado a uma equação; número associado ao modo de

n, vibração;

$n_{h}$, parâmetro de rigidez para solos coesivos;

$s, \quad$ coordenada ao longo do eixo deformado;

$t$ tempo;

u, deslocamento axial da estaca;

$x$, coordenada axial;

$z$, profundidade a qual está inserida a estaca;

w, deslocamento lateral da estaca;

$w_{1}$, deslocamento lateral da sub-coluna 1, desenterrada;

$w_{2}$, deslocamento lateral da sub-coluna 2, enterrada;

$w, \quad$ derivada do deslocamento $w$ em relação ao eixo x, ou seja, $\frac{d w}{d x} ;$

$\bar{w}$, parâmetro adimensional do deslocamento lateral da coluna; 
$\bar{w}^{\prime}, \quad$ derivada do parâmetro adimensional do deslocamento lateral da coluna em relação a $\zeta$;

$\alpha_{i}, \quad$ raiz do polinômio característico;

$\beta_{1}, \quad$ parâmetro de amortecimento;

$\delta$, variação de termos;

$\phi$, ângulo de fase;

$\phi_{\mathrm{j}}$, função em seno constituinte da função de aproximação $f_{n}$;

$\lambda$, parâmetro de carga adimensional;

$\pi$, energia potencial total;

$\rho, \quad$ massa específica por unidade de comprimento;

$\omega_{0}, \quad$ freqüência natural;

$\xi$ fator de amortecimento, variável de integração;

$\psi$, ângulo formado entre o eixo x e o eixo da viga;

$\zeta, \quad$ parâmetro adimensional da coordenada axial;

$\Delta, \quad$ encurtamento da extremidade da estrutura;

$\Omega, \quad$ parâmetro adimensional de freqüência;

$\Omega_{\mathrm{n}}, \quad$ parâmetro da freqüência natural;

$\in, \quad$ erro associado ao método de Newton-Rapson. 